\title{
Solitary prostatic cancer metastasis to the testis: A case report and lessons to learn
}

\author{
Asmaa Ismail, Hazem Elmansy, Walid Shahrour, Owen Prowse, Ahmed Kotb \\ Urology Department, Northern Ontario School of Medicine, Thunder Bay, Ontario, Canada.
}

\begin{abstract}
Summary Prostate cancer (Pca) is a complex disease. Several case series and reports have described the spread of Pca to unusual organs, like esophagus, eye and periureteric fat causing ureteropelvic junction obstruction. Spread of Pca to the testis has been reported in few case reports, however Pca was always firstly diagnosed in all published cases and testicular spread of cancer has been diagnosed during follow up of the patients. This case is unique in that, the patient initially presented with a testicular mass and histologic examination after orchiectomy allowed to diagnose prostatic cancer. This patient was 81 years old and he never had PSA screening by his family doctor. PSA was not even done initially by us considering his age and the presentation with testicular mass. This case may impact clinical practice in several ways: 1) considering Pca always in the differential diagnosis of any disease of an adult man, regardless of its presentation because we did not do that at initial patient evaluation and PSA was only measured after orchiectomy when pathology demonstrated metastatic Pca; 2) suggesting standard orchiectomy with epididemectomy for surgical castration instead of the current surgical technique of subcapsular/subepididymal orchiectomy, because our patient had cancer involving his epididymis as well; 3) suggesting to include PSMA as a part of preoperative staging for high risk Pca patients, in consideration that PSMA is proving to be a promising new imaging technique that can help diagnosing metastatic Pca in unusual locations.
\end{abstract}

KEY WORDS: Prostate cancer; Testicular metastasis; PSMA; Orchiectomy.

Submitted 17 August 2019; Accepted 1 September 2019

\section{INTRODUCTION}

Prostate cancer (Pca) is a complicated disease that should be considered in the differential diagnosis of any disease in a man over 50 year old, regardless of his initial presentation. Pastore et al. (1) recently published a case of Pca presented with ocular symptoms turned to be orbital metastasis. Mittal et al. (2) described a patient that had bilateral orchiectomy for hormonal control of metastatic Pca where histology demonstrated metastatic Pca of the testis and epididymis.

\section{Case report}

An 81- years old man presented to the urology clinic with a left hard and painless testicular mass. Tumour markers were normal and CT of chest, abdomen and pelvis was normal. He was counseled and left radical orchiectomy was done. Pathology was metastatic adenocarcinoma likely prostatic in origin. The tumour mainly involved epididymis and lower half of the testis. The tumour did show positive immunohistochemical staining to pan-keratin, EMA, CK8/18, p53, BerEP4 and PSA, but negative for CD30, CK7, CK20, alpha-fetoprotein, placental alkaline phosphatase, hCG, CEA, vimentin, calretinin, TTF-1, and CK 5/6. There was no previous PSA testing done. PSA was then measured and transrectal ultrasound (TRUS) guided biopsy was scheduled. PSA was $66 \mathrm{ng} / \mathrm{ml}$ and TRUS guided biopsy confirmed Pca Gleason $4+4$ in all cores. Bone scan was negative. The patient was counseled about his diagnosis and the absence of other evidence of metastases. External radiotherapy was started with a curative intent.

\section{Discussion AND CONCLUSIONS}

Almagro et al. (3) had the first PubMed indexed publication describing accidentally discovered Pca in the testis during bilateral orchiectomy done for hormonal control. Since then; infrequent case reports are being published describing the testicular spread of Pca. However, all available case reports included patients known to have Pca and had testicular metastasis during follow up course of cancer. Table 1 summarizes ways of presentation of testicular metastasis from Pca.

This case report is unique as it describes a metastatic testicular lesion as a way to diagnose Pca. It even leads us to few questions that should be considered during management of this complex disease. Is subcapsular/subepididymal orchiectomy a valid option for surgical castration, considering the risk of epididymal silent metastasis? This

\section{Table 1.}

Some studies of patients with Pca presenting with testicular mass.

\begin{tabular}{|ll|}
\hline Publications & Presentation \\
\hline Janssen et al. (4) & During follow up after radical prostatectomy \\
Menchini-Fabris et al. (5) & \\
\hline Campara et al. (6) & During hormonal treatment of metastatic Pca \\
Kusaka et al. (7) & \\
\hline Mittal et al. (2) & As a part of initial management of metastatic Pca \\
Almagro et al. (3) & \\
\hline This case report & Metastatic Pca to the testis without previous Pca diagnosis \\
\hline
\end{tabular}


patient had his cancer involving both the testis and epididymis with no clinical suspicion for epididymal involvement. Santos-Lopes et al. (8) described a case of Pca developing epididymal metastasis during follow up of Pca that was discovered because of rising PSA. Are CT bone scans sufficient for patients presenting with high risk criteria of Pca? A recent case series (9) showed that PSMA was able to detect metastatic Pca in unusual sites. Another study included 21 patients with high risk Pca proved superiority of PSMA over CT scan, bone scan and MRI, with PSMA changing management of $51 \%$ of patients (10).

Prostate cancer should be always suspected in disease of adult men regardless of their presentation. Subcapsular/ subepididymal orchiectomy may not be a safe option for surgical castration. PSMA scan may be the future single routine investigation for all patients with high risk prostate cancer.

\section{References}

1. Pastore MR, D'Aloisio R, Cirigliano G, et al. Orbital metastasis as presenting symptom from a prostatic adenocarcinoma. Eur J Ophthalmol. 2019; 1120672119832182.

2. Mittal J, Dorairajan LN, Manikandan R, et al. Testicular and epididymal metastasis from prostate carcinoma: a rare manifestation of common disease. J Clin Diagn Res. 2017; 11:PD01- PD02.
3. Almagro UA. Metastatic tumors involving testis. Urology. 1988; 32:357-60.

4. Janssen S, Bernhards J, Anastasiadis AG, et al. Solitary testicular metastasis from prostate cancer: a rare case of isolated recurrence after radical prostatectomy. Anticancer Res. 2010; 30:1747-9.

5. Menchini-Fabris F, Giannarini G, Pomara G, et al. Testicular metastasis as isolated recurrence after radical prostatectomy. A first case. Int J Impot Res. 2007; 19: 108-9.

6. Campara Z, Simic D, Aleksic P, et al. Metastasis of prostate adenocarcinoma to the testis. Med Arch. 2016; 70:318- 20.

7. Kusaka A, Koie T, Yamamoto H, et al. Testicular metastasis of prostate cancer: a case report. Case Rep Oncol. 2014; 7:643-7.

8. Santos-Lopes S, Lobo J, Henrique R, et al. Epididymal metastasis from prostate adenocarcinoma: an unusual and challenging diagnosissuspected in gallium-68 prostate-specific membrane antigen-positron emissiontomography/computed tomography and histologically confirmed. Urol Ann. 2017; 9:89-91.

9. Dureja S, Thakral P, Pant V, et al. Rare sites of metastases in prostate cancer detected on Ga-68 PSMA PET/CT scan. A case series. Indian J Nucl Med. 2017; 32:13-15.

10. Hirmas N, Al-Ibraheem A, Herrmann K, et al. [68Ga]PSMA PET/CT improves initial staging and management plan of patients with high-risk prostate cancer. Mol Imaging Biol. 2019; 21:574-581.

\section{Correspondence}

Asmaa Ismail, MD

asmaaismail@rocketmail.com

Hazem Elmansy, MD

hazemurol00@yahoo.com

Walid Shahrour, MD

walid.shahrour@gmail.com

Owen Prowse, MD

owenprowse@rogers.com

Ahmed Kotb, MD

drahmedfali@gmail.com

Urology Department, Northern Ontario School of Medicine,

Thunder Bay Regional Health Sciences Centre, 980 Oliver Rd,

Thunder Bay, Ontario P7B 6V4 (Canada) 